\title{
NOTES D'ACTUALITÉ
}

\section{À bout de souffle : la santé mentale des travailleurs essentiels sous la loupe}

\author{
Landry Kuate ${ }^{a}$, Salmata Ouedrago ${ }^{b}$
}

\section{Introduction}

Cet article analyse les potentiels impacts ou les conséquences de la pandémie de COVID-19 sur les travailleurs de la santé. Cette pandémie constitue depuis maintenant plus d'un an l'un des principaux problèmes de santé publique de la dernière décennie, causant d'importantes conséquences macroéconomiques et microéconomiques, aussi bien à l'échelle de la population que de l'ensemble du pays. Sur le plan macroéconomique, on peut citer entre autres l'augmentation du taux de chômage, de l'inflation et de la dette et, au contraire, la baisse des recettes due au ralentissement de l'activité économique. Sur le plan microéconomique, la pandémie affecte la santé globale des individus et la productivité des travailleurs, et exacerbe toutes les inégalités socioéconomiques.

Au Canada, bien que les systèmes de santé provinciaux soient modernes et efficaces, il demeure que les répercussions de la pandémie sont remarquables et probantes. Par exemple, les travailleurs de la santé, considérés comme des travailleurs essentiels, sont en première ligne de la stratégie de riposte contre la pandémie. Ils font face à des défis particuliers et majeurs. Les conditions de travail exigeantes, le risque accru d'infection, le taux de mortalité élevé, le niveau de stress de la charge de travail élevée et des problèmes d'approvisionnement en équipements de protection affectent la santé mentale de ces travailleurs. De plus, comme la majorité de la population, ils sont exposés à un nombre considérable de fausses nouvelles et de rumeurs qui augmentent leur anxiété.

Une étude réalisée par Statistique Canada auprès de 18000 travailleurs de la santé en novembre et décembre 2020 révèle que $70 \%$ d'entre eux ont une moins bonne santé mentale par rapport à mars 2020 . L'étude démontre aussi que les problèmes d'approvisionnement en équipements de protection contribuent à augmenter le niveau de stress de ces travailleurs. Ce contexte de travail augmente les risques de maladies psychologiques ainsi que la détresse physique et émotionnelle chez le personnel médical. Cette pression énorme sur les travailleurs de la santé et sur le système de santé a d'importants coûts socioéconomiques directs et indirects (p. ex., augmentation de la demande de services médicaux, baisse de la productivité et perte de qualité de vie). La figure ci-après résume de façon non exhaustive quelques conséquences socioéconomiques importantes de la pandémie. De façon spécifique, notre analyse met l'accent sur le cas des travailleurs essentiels.

\footnotetext{
a Doctorant en économie, Université d'Ottawa

${ }^{\mathrm{b}}$ Professeure, Université du Québec à Chicoutimi 


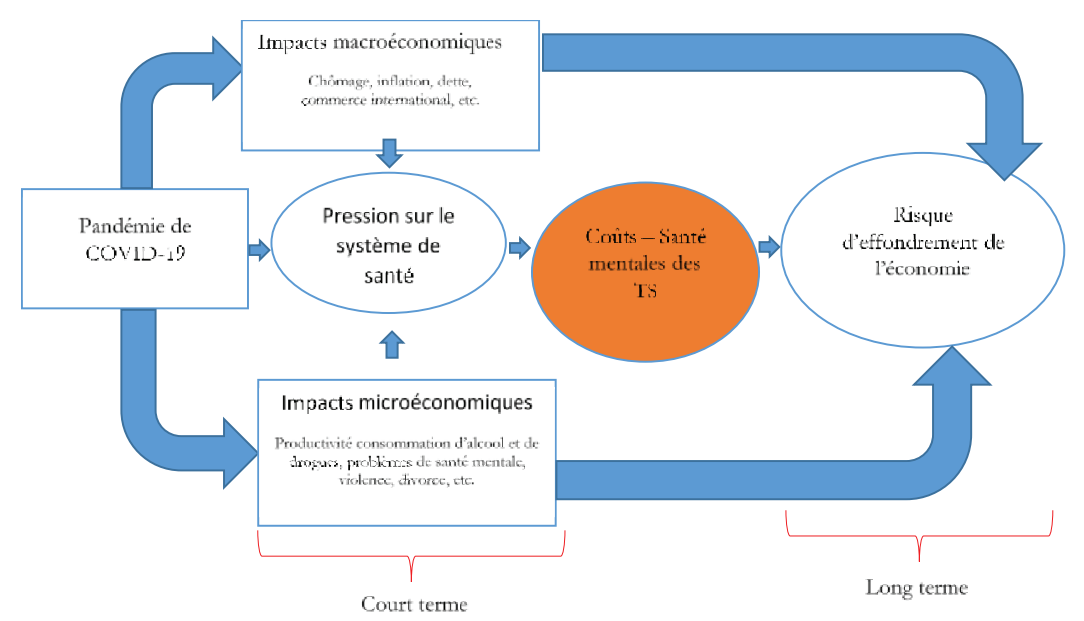

Figure 1 - Conséquences socioéconomiques de la pandémie

Source : Landry et Ouedraogo, 2021

Dans l'hypothèse de l'absence de mesures ciblées et efficaces pour soutenir le système de santé et surtout les travailleurs essentiels, les perspectives de reprise économique seront mitigées.

Les interventions pour réduire la prévalence de la santé mentale chę les travailleurs de la santé: ce qu'en dit la littérature

La pandémie de COVID-19 provoque une forte occurrence ou prévalence des problèmes de santé mentale chez les travailleurs de la santé. Cette situation découle du fait que la plupart des travailleurs évoluent dans des services isolés sans recevoir de formation adéquate pour améliorer ou maintenir leur santé mentale. Par conséquent, des soins psychologiques réguliers sont nécessaires pour répondre à ces besoins, surtout en période de crise sociosanitaire. La littérature présente quelques interventions et/ou solutions qui permettraient de minimiser les impacts négatifs, aussi bien pour les travailleurs que pour l'ensemble de l'économie. Entre autres interventions, nous pouvons citer i) les interventions de protection, de soutien direct, d'encouragement et de motivation; ii) les programmes de formation; et iii) la télémédecine.

\section{Conclusion}

Dans un contexte de vieillissement de la population, ces solutions sont non seulement bénéfiques pour faire face à la pandémie actuelle de COVID-19, mais aussi envisagées pour les futures épidémies de maladies infectieuses et/ou quand le système de santé est sous pression. Bien que ces mesures et interventions soient nécessaires pour aider à prévenir ou à prendre en charge les problèmes de santé mentale chez les travailleurs de la santé, il n'est pas exclu que cette problématique soit endogène à la perception que les travailleurs ont de la valorisation ou de la reconnaissance de leur profession. Dans ce cas, il serait préférable et pertinent de fournir des solutions non financières qui ciblent particulièrement ces aspects, par exemple la mise en place de stratégies visant à améliorer la reconnaissance de l'utilité sociale des travailleurs de la santé.

\section{RÉFÉRENCES}

Chen, Q., Liang, M., Li, Y., Guo, J., Fei, D., Wang, L. et collab. (2020). Mental health care for medical staff in China during the COVID-19 outbreak. The Lancet Psychiatry, 7(4), e15-e16. https://doi.org/10.1016/S2215-0366(20)30078-X

Lai, J., Ma, S., Wang, Y., Cai, Z., Hu, J., Wei, N., Wu, J., Du, H., Chen, T., Li, R., Tan, H., Kang, L., Yao, L., Huang, M., Wang, H., Wang, G., Liu, Z. et Hu, S. (2020). Factors associated with mental health outcomes among health care workers exposed to coronavirus disease 2019. JAMA Network Open, 3(3), e203976. https:/ /doi.org/10.1001/jamanetworkopen.2020.3976

Schwartz, J., King, C.-C. et Yen, M.-Y. (2020). Protecting healthcare workers during the coronavirus disease 2019 (COVID-19) outbreak: Lessons from Taiwan's severe acute respiratory syndrome response. Clinical Infectious Diseases, 71(15), 858-860. https://doi.org/10.1093/cid/ciaa255

Xiao, H., Zhang, Y., Kong, D., Li, S. et Yang, N. (2020). The effects of social support on sleep quality of medical staff treating patients with coronavirus disease 2019 (COVID-19) in January and February 2020 in China. Medical Science Monitor: International Medical Journal of Experimental and Clinical Research. 26:e923549. https://doi.org/10.12659/msm.923549 